\title{
The Influence of Phase-Modulation on Femtosecond Time-Resolved Coherent Raman Spectroscopy
}

\author{
W. Holzapfel, R. Leonhardt, and W. Zinth \\ Physik Department, Technische Universität, D-8000 München, Fed. Rep. Germany
}

Received 25 April 1988/Accepted 2 August 1988

\begin{abstract}
The influence of phase-modulation on femtosecond time-resolved coherent Raman scattering is investigated theoretically and experimentally. The coherent Raman signal taken as a function of the spectral position shows unexpected temporal oscillations close to time zero. A theoretical analysis of the coherent Raman scattering process indicates that the femtosecond light pulses are amplitude and phase modulated. The pulses are asymmetric in time with more slowly decaying trailing wings. The phase of the pulse amplitude contains quadratic and higher-order contributions.
\end{abstract}

PACS: $42.65,42.80$

Since the advent of the colliding-pulse mode-locking in 1981 light pulses shorter than $100 \mathrm{fs}$ have found extensive application in the study of ultrafast phenomena [1]. Various novel laser techniques have been developed to generate intense and reproducible femtosecond light pulses [2-4]. The different laser systems show interesting common features: (i) the shortest pulses are generated in the red wing of the gain and absorption spectrum of the amplifying and modelocking dyes. (ii) The dispersion in the resonator has to be compensated carefully. (iii) The spectra of the light pulses usually are asymmetric showing a steeper decay in the red part of the spectrum. The latter indicates that the light pulses are phase-modulated. Phase modulation or chirp have been discussed since the first publications on colliding-pulse mode-locked lasers $[1,5]$. Direct experimental determination of phase modulation of femtosecond light pulses was performed recently by numerical deconvolution or fitting of measured correlation traces [6-9]. The significance of the phase modulation on the results of ultrafast experiments was addressed by Fujimoto et al. [10], where the influence of phase-modulation on a timeresolved transmission experiment was demonstrated.

In this paper we investigate the effect of phasemodulation on time-resolved coherent Raman scattering. The experimental data show unexpected modul- ations close to time zero, which can be explained by certain amplitude and phase properties of the femtosecond light pulses.

\section{Theory of Time-Resolved Coherent Raman Scattering}

The principle of time-resolved coherent Raman scattering has been treated in a number of publications $[11,12]$. At time zero two short light pulses at the laser and the Stokes frequency (center frequencies $\Omega_{L}$ and $\Omega_{S}$, respectively) enter the sample and excite a molecular vibration at the frequency $\Omega_{q}$ via transient stimulated Raman scattering. The light fields are written as plane waves, e.g., $E_{L}=\frac{1}{2} E_{L}(t) \exp \left(-\mathrm{i} \Omega_{L} t\right)$ + c.c., where phase-modulation is contained in the complex function $E_{L}(t)$. In our experiments the laser pulses are much shorter than the Stokes pulses and the time dependence of the driving force $F \propto E_{L}(t) E_{S}^{*}(t)$ is only determined by the laser field, $F(t)=E_{L}(t) E_{S}^{*}$. $E_{S}^{*}$ is the effective Stokes amplitude averaged over the duration of the laser pulse. The coherent vibrational excitation is represented by the coherent amplitude $\langle q\rangle=\mathrm{i} / 2 Q(t) \exp \left(-\mathrm{i} \Omega_{q} t\right)+$ c.c. $:$

$Q(t)=\kappa_{2} \int_{-\infty}^{t} d t^{\prime} E_{L}\left(t^{\prime}\right) E_{S}^{*} \exp \left[\left(t^{\prime}-t\right) / T_{2}-\mathrm{i} \Delta \omega t^{\prime}\right]$. 
The parameter $\kappa_{2}$ contains material parameters like the Raman cross section, it was defined in [11]. $T_{2}$ is the dephasing time of the vibration. We assume $T_{2}$ to be much longer than the duration $t_{p}$ of the laser pulses, $T_{2} \gg t_{p} . \Delta \omega$ is the frequency mismatch between the frequency of the driving force $\Omega_{F}=\Omega_{L}-\Omega_{S}$ and the molecular frequency $\Omega_{q}, \Delta \omega=\Omega_{L}-\Omega_{S}-\Omega_{q}$. The time dependence of the coherent amplitude $\langle q\rangle$ is monitored by coherent Raman scattering of a third probing light pulse $E_{p}=\frac{1}{2} E_{p}(t) \exp \left(-\mathrm{i} \Omega_{p} t\right)+$ c.c. In the experiment we use a probing pulse which is derived from the exciting laser pulse by means of a beam splitter. Therefore, we may write $\Omega_{p}=\Omega_{L}$ and $E_{p}(t)=E_{L}\left(t-t_{D}\right)$. In standard time resolved coherent Raman experiments the coherently scattered energy is measured as a function of the time delay $t_{D}$ between the exciting and probing pulses. It was shown previously [13] that interesting details of the molecular transition are revealed when the spectrum of the coherently scattered light, e.g., the anti-Stokes part of the spectrum, is detected. The signal energy at the frequency position $\omega_{A}=2 \Omega_{L}-\Omega_{S}+\Delta \omega_{A}$ becomes

$S\left(t_{D}, \Delta \omega, \Delta \omega_{A}\right) \propto\left|\int d t^{\prime} E_{A}\left(t_{D}, t^{\prime}, \Delta \omega\right) \exp \left(\mathrm{i} \Delta \omega_{A} t^{\prime}\right)\right|^{2}$

where

$$
\begin{aligned}
& E_{A}\left(t_{D}, t^{\prime}, \Delta \omega\right) \\
& \propto E_{p}\left(t^{\prime}-t_{D}\right)\left(\int _ { - \infty } ^ { t ^ { \prime } } d t ^ { \prime \prime } \left\{\exp \left[-\left(t^{\prime}-t^{\prime \prime}\right) / T_{2}\right]\right.\right. \\
& \left.\quad \times \exp \left[\mathrm{i} \Delta \omega\left(t^{\prime}-t^{\prime \prime}\right) E_{L}\left(t^{\prime \prime}\right)\right]\right\} \\
& \left.-3 / 2 \frac{\mathrm{i} \chi_{\mathrm{NR}}^{(3)}}{\chi_{\mathrm{RES}}^{(3)}} E_{L}\left(t^{\prime}\right)\right) .
\end{aligned}
$$

Equation (3) was calculated using the polarization geometry of our experiments where $E_{L}$ and $E_{S}$ are parallel polarized while $E_{P}$ and $E_{A}$ are polarized perpendicular to $E_{L}$.

The signal $S$ is the absolute square of the Fourier transform of the coherently scattered anti-Stokes field $E_{A}$, which is determined by scattering via the resonant coherent excitation $Q$, first part of (3), and via the nonresonant nonlinear susceptibility $\chi_{\mathrm{NR}}^{(3)}$ of the sample. $\chi_{\mathrm{RES}}^{(3)}$ is the resonant nonlinear susceptibility containing molecular parameters like Raman crosssection and dephasing time. $\chi_{\mathrm{RES}}^{(3)}$ was defined in [14]. The measured anti-Stokes signal $S\left(t_{D}, \Delta \omega, \Delta \omega_{A}\right)$ depends on three parameters: the delay time $t_{D}$, the frequency mismatch $\Delta \omega$ of the excitation which may be adjusted via the Stokes frequency $\Omega_{S}$, and the frequency position $\Delta \omega_{A}$ of the spectrometer. For the following analysis we rewrite the coherent signal as a convolution of an experimental "excite and probe" function $f\left(t, \Delta \omega_{A}\right)$ and the "coherent response" func- tion $r\left(t, \Delta \omega+\Delta \omega_{A}\right)$ :

$$
\begin{aligned}
& S\left(t_{D}, \Delta \omega, \Delta \omega_{A}\right) \\
& \quad \propto\left|\int_{0}^{\infty} d \operatorname{tr}\left(t, \Delta \omega+\Delta \omega_{A}\right) f\left(t_{D}-t, \Delta \omega_{A}\right)\right|^{2}
\end{aligned}
$$

with

$$
\begin{gathered}
f\left(t, \Delta \omega_{A}\right)=\int_{-\infty}^{+\infty} d t^{\prime} \exp \left(\mathrm{i} \Delta \omega_{A} t^{\prime}\right) E_{L}\left(t^{\prime}\right) E_{P}\left(t^{\prime}-t\right), \\
r\left(t, \Delta \omega+\Delta \omega_{A}\right)=\exp \left(-t / T_{2}\right) \exp \left[\mathrm{i}\left(\Delta \omega+\Delta \omega_{A}\right) t\right] \\
-3 / 2 \frac{\mathrm{i} \chi_{\mathrm{NR}}^{(3)}}{\chi_{\mathrm{RES}}^{(3)}} \delta\left(t / T_{2}\right) .
\end{gathered}
$$

The "coherent response function" $r\left(t, \Delta \omega+\Delta \omega_{A}\right)$ contains the influence of the investigated material. The first part describes the slow reaction of the resonantly excited vibration determined by the dephasing time $T_{2}$. The nonresonant susceptibility $\chi_{\mathrm{NR}}^{(3)}$ has an instantaneous response. Its magnitude can be determined in a steady-state coherent anti-Stokes measurement [15].

Of special interest is the "excite and probe" function $f\left(t, \Delta \omega_{A}\right)$, which is the Fourier transform of the product of exciting and probing light pulse. Its absolute square may also be measured in an independent experiment: One obtains $\left|f\left(t_{D}, \Delta \omega_{A}\right)\right|^{2}$ when the spectrum of the intensity autocorrelation function $A\left(t_{D}\right)$ (measured via background-free sum frequency generation) is investigated [9]:

$A\left(t_{D}, 2 \Omega_{L}+\Delta \omega_{L}\right) \propto\left|f\left(t_{D}, \Delta \omega_{A}\right)\right|^{2}$.

This function is also obtained in a coherent Raman experiment when probing a purely nonresonant nonlinear susceptibility $\chi_{\mathrm{NR}}^{(3)}$, i.e., in experiments where the first part on the rhs of (6) may be neglected.

Equations (4-7) are used below to simulate the results of the coherent Raman experiment. Via (7) we determine the absolute square of the excited and probe function $f\left(t, \Delta \omega_{A}\right)$, while a least square fitting procedure for $S\left(t_{D}, \Delta \omega, \Delta \omega_{A}\right)$ is used to determine the phase of the complex function $f\left(t, \Delta \omega_{A}\right)$. From the latter the amplitude and phase of the laser pulses $E_{p}$ will be obtained by Fourier analysis of (5).

\section{Experimental}

The basic features of the experimental system are as follows [16]: Picosecond pulses from a mode-locked argon-ion laser synchronously pump two dye lasers. The Stokes pulses are generated in a standard synchronously mode-locked picosecond dye laser (dye DCM) with a three-plate birefringent filter providing a tunability at the Stokes frequency of our experiment between 15,600 and $14,500 \mathrm{~cm}^{-1}$. The Stokes pulses have a duration of $6 \mathrm{ps}$ and a spectral width of $3 \mathrm{~cm}^{-1}$. 
The exciting and probing laser pulses are obtained from an uni-directional ring (UDR) dye laser. This laser may generate femtosecond pulses as short as $32 \mathrm{fs}$ $(\lambda \cong 620 \mathrm{~nm})$. In the experiments the laser was operated in a regime of high pulse stability yielding pulse durations of approximately $85 \mathrm{fs}$. Details of the measurement of the pulse duration are discussed below. The spectrum of the femtosecond pulses is broad, $\Delta v \cong 135 \mathrm{~cm}^{-1}$. It is analyzed in detail below. Part of the femtosecond pulses from the UDR laser $\left(E_{L}\right)$ and the Stokes pulses $\left(E_{S}\right)$, both with parallel polarisation, excite the molecular vibration in the sample. The coherent vibrational excitation is monitored by the delayed probing pulses $\left(E_{P}\right)$ deduced from the exciting laser pulse $E_{L}$ by a beam splitter. The probing pulses are polarized perpendicular to the exciting pulses. Anti-Stokes light (polarized parallel to the probing pulse) generated by the probing process is analyzed by a $10 \mathrm{~cm}$ grating spectrometer with a bandwidth of $40 \mathrm{~cm}^{-1}(1.3 \mathrm{~nm})$.

For the measurement of the background-free autocorrelation function the sample was replaced by a $100 \mu \mathrm{m}$ thick KDP crystal and light at the sum frequency was detected after passing through a broadband filter (standard autocorrelation measurement) or a spectrometer with a bandwidth of $50 \mathrm{~cm}^{-1}$ (for the frequency resolved auto-correlation measurement).

\section{Results and Discussion}

\subsection{The Spectrum and the Autocorrelation Function of the Femtosecond Laser Pulses}

The emission spectrum of the uni-directional ring dye laser is shown in Fig. 1. The frequency position

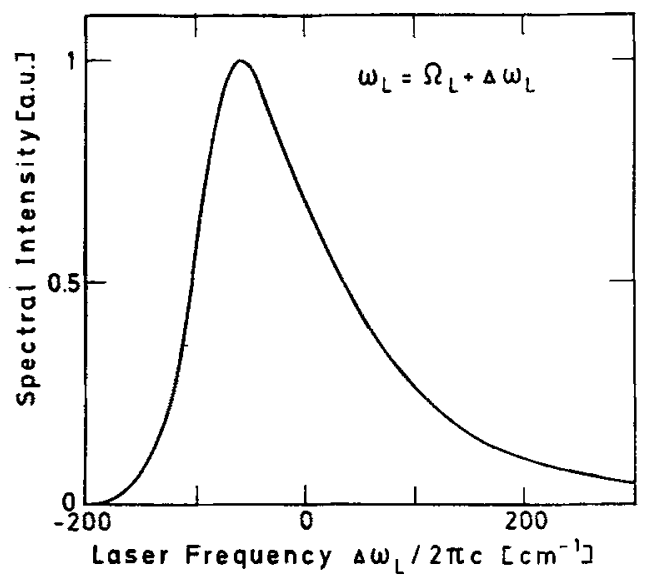

Fig. 1. Spectrum of the dye-laser pulses from the synchronously pumped UDR laser. From the spectral width of $135 \mathrm{~cm}^{-1}$ and a pulse duration of $85 \mathrm{fs}$ one deduces the bandwidth product to be $\Delta \omega t_{p}=0.34$ corresponds to $\Omega_{L} / 2 \pi c=15,980 \mathrm{~cm}^{-1}$. The halfwidth (FWHM) of the spectrum is $\Delta v / c=135 \mathrm{~cm}^{-1}$, which together with the pulse duration of $85 \mathrm{fs}$ (deduced from the autocorrelation function and the assumption of sech-shaped pulses) - gives a bandwidth product of $\Delta v t_{p}=0.34$. This value is close to the theoretical value of $\Delta v t_{p}=0.315$ for sech-shaped pulses and would suggest nearly bandwidth-limited pulses. The pulse spectrum, however, shows an asymmetry with rapidly decaying red wings and slowly decaying blue wings, which indicates phase-modulation and/or an asymmetric pulse envelope.

Due to the special shape of the spectrum we cannot directly deduce from Fig. 1 the carrier frequency $\Omega_{L}$ of the laser pulses. For this reason we measured the spectrum of the frequency-doubled laser pulses. This spectrum is nearly symmetric peaking at $\omega_{2 \mathrm{H}} / 2 \pi c$ $=31.960 \mathrm{~cm}^{-1}$. From this value we define the carrier frequency of the fundamental pulses to be $\Omega_{L}=\omega_{2 \mathrm{H}} / 2$; $\Omega_{L} / 2 \pi c=15.980 \mathrm{~cm}^{-1}$. This value is consistent with the frequency obtained by coherent scattering from a purely nonresonant susceptibility. Here the spectrum of the anti-Stokes light was found to be nearly symmetric with a peak at $\omega_{\mathrm{NR}}=2 \Omega_{L}-\Omega_{S}$. We conclude that the central frequency components of the laser spectrum which are responsible for both, most efficient second-harmonic frequency generation and coherent Raman scattering, are at the "center frequency" $\Omega_{L}$. Note that this frequency is not at the peak of the laser spectrum of Fig. 1 but is blue-shifted by $60 \mathrm{~cm}^{-1}$.

A further indication for phase-modulation and deviation from the sech-shape comes from the measurement of the spectrally resolved autocorrelation function [9]. In Fig. 2 (broken lines, circles) the

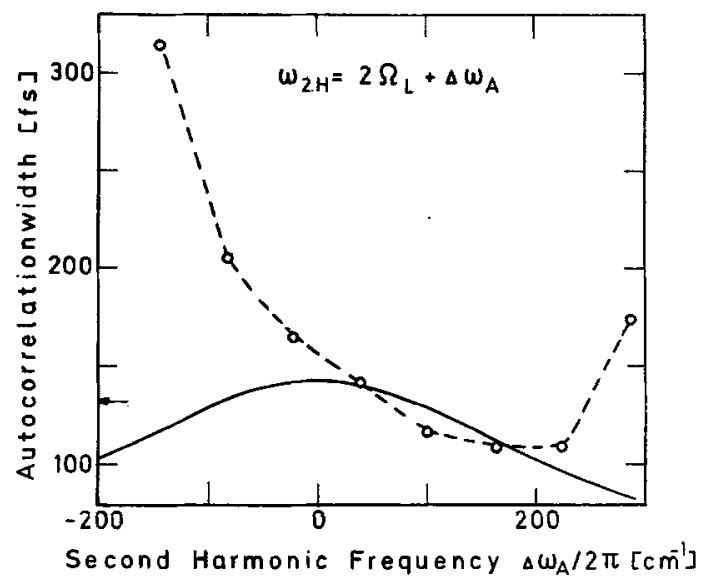

Fig. 2. Measured width (points) of the spectrally resolved autocorrelation function plotted as a function of the second harmonic frequency $\Delta \omega_{A}$. The solid curve is calculated for a sech-shaped pulse with pulse duration of $85 \mathrm{fs}$. The arrow indicates the width obtained for a conventional (spectrally unresolved) autocorrelation measurement 
halfwidth of the autocorrelation $t_{A}$ is plotted as a function of the detection frequency. While very small autocorrelation widths of $\Delta t_{A} \cong 100 \mathrm{fs}$ are found in the middle- and high-frequency part of the secondharmonic spectrum, they considerably increase in the low- and very high-frequency wings up to values of $300 \mathrm{fs}$. This is in clear contradiction to the solid curve in Fig. 2 which represents the calculated results for sech-shaped - not phase-modulated - pulses of $85 \mathrm{fs}$ duration. For these bandwidth-limited pulses a spectrally unresolved autocorrelation width of $130 \mathrm{fs}$ results as indicated by the arrow.

\subsection{Time Resolved Coherent Raman Scattering of the $992 \mathrm{~cm}^{-1}$ Mode of Benzene}

We present here the experimental investigations of coherent Raman scattering performed for liquid benzene. We investigated the symmetric $v_{1}$ mode of benzene at $992 \mathrm{~cm}^{-1}$ which has a high Raman crosssection, is strongly polarized, and where the ratio $\chi_{\mathrm{NR}}^{(3)} \chi_{\mathrm{RES}}^{(3)} \cong 10^{-2}$ may be calculated from literature data $[11,15]$. The dephasing time $T_{2}=4.7 \mathrm{ps}$ of this mode is approximately fifty times longer than the laser pulses used in our experiments. The results of a series of experiments on this benzene mode are summarized as follows:

a) With a broad-band detection system and a resonant excitation $\Omega_{L}-\Omega_{S}=\Omega_{q}$ the observations are qualitatively described by the theory given above [11, 12]. A rapid rise of the signal and an exponential decay at later delay times is found. In order to describe the results theoretically we applied a "simplified numerical model". We used the molecular parameters as dephasing time $T_{2}$ and $\chi_{\mathrm{NR}}^{(3)} / \chi_{\mathrm{RES}}^{(3)}$ and assumed that the laser pulses were coherent and sech-shaped without phasemodulation. With this "simplified model" we could readily describe the experimental results besides the fact that the signal peak in the experiment occurs later in time than expected from the calculation.

b) After detuning of the excitation an overshoot of the signal is observed at time zero which is larger than the overshoot calculated with the simplified numerical model.

c) Narrow-band detection of the anti-Stokes signal (bandwidth of $40 \mathrm{~cm}^{-1}$ ) combined with resonant excitation again yields a slightly delayed rise of the coherent signal.

d) The strongest deviations of the measured coherent signal from the results of the "simplified numerical model" are found for narrow-band detection and a detuning of the excitation frequency. The experimental results obtained under these experimental conditions are depicted in Fig. 3. Here we plotted the normalized coherent signal as a function of the delay time for different settings of the spectrometer from $\omega_{A} / 2 \pi c$

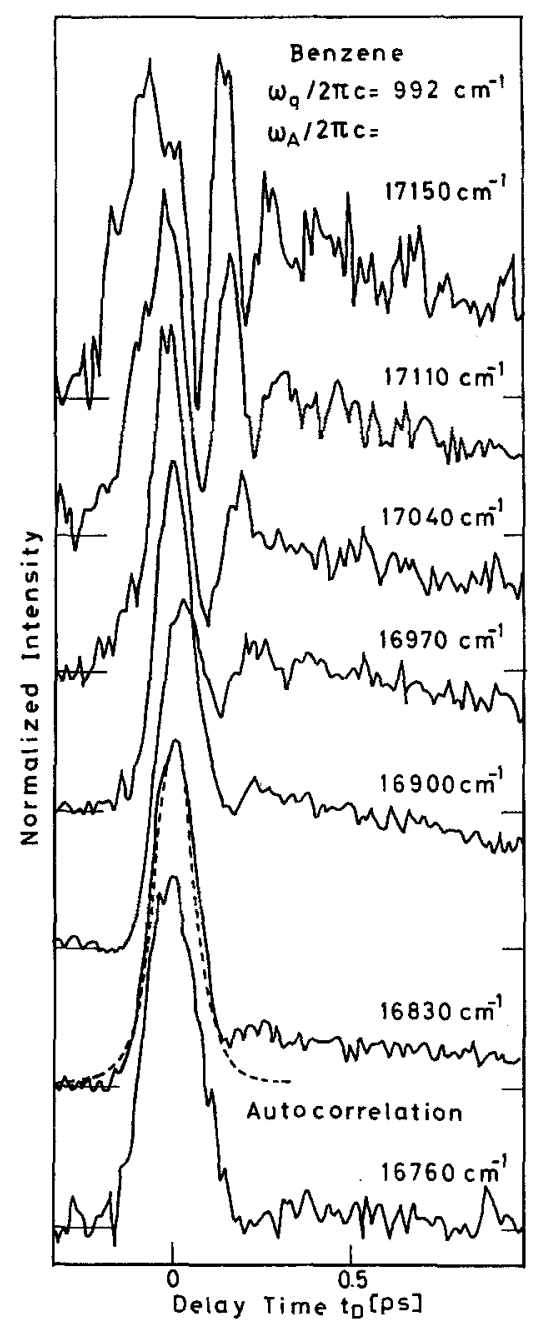

Fig. 3. Time resolved coherent Raman scattering after excitation of the $v_{1}$ mode of benzene at $992 \mathrm{~cm}^{-1}$. The anti-Stokes signal is detected as a function of time for various anti-Stokes frequencies $\omega_{A}$

$=17.150 \mathrm{~cm}^{-1}$ to $16.760 \mathrm{~cm}^{-1}$. The Stokes frequency was at $\Omega_{\mathrm{S}} / 2 \pi c=15.060 \mathrm{~cm}^{-1}$ and the laser frequency at $\Omega_{L} / 2 \pi c=15.980 \mathrm{~cm}^{-1}$. We estimate the detuning of the excitation force to be approximately $\Delta \omega / 2 \pi \mathrm{c}$ $=-70 \mathrm{~cm}^{-1}$.

The main features of the signal curve are as follows: At small anti-Stokes frequencies, e.g., $16.760 \mathrm{~cm}^{-1}$ and $16.830 \mathrm{~cm}^{-1}$, the nonresonant part of the signal dominates: At time zero a pronounced peak is found which follows the autocorrelation trace (broken curve). With increasing frequencies the resonant contribution to the signal (at later delay times) becomes more important and a dip shows up at a delay time of $t_{D} \cong 200 \mathrm{fs}$ $\left(\omega_{A} / 2 \pi c=16.900 \mathrm{~cm}^{-1}, 16.970 \mathrm{~cm}^{-1}\right)$. Note that only the early part of the exponential decay of the signal is shown in Fig. 3. For larger anti-Stokes frequencies, $\omega_{A} / 2 \pi c>17.040 \mathrm{~cm}^{-1}$ a pronounced beating with beat periods of $140 \mathrm{fs}$ or a beat frequency of $\cong 240 \mathrm{~cm}^{-1}$ appears, which persist up to $t_{D} \cong 300 \mathrm{fs}$. 


\subsection{Numerical Analysis of the Experimental Data}

In a first approach we tried to describe the experimental results of Fig. 3 using sech-shaped pulses without phase-modulation. We used a variable ratio of $\chi_{\mathrm{NR}}^{(3)} / \chi_{\mathrm{RES}}^{(3)}$ and we expected changes of the coherent response function due to the collision process which may be important at earlier delay times (Kubo's lineshape theory) [12]. Even varying the different parameters over a wide range did not allow to describe the experiment qualitatively.

In order to obtain a consistent description of the experimental results we introduced phase-modulated laser pulses with asymmetric amplitudes. These pulses lead to a complex "excite and probe" function $f\left(t_{D}, \Delta \omega_{A}\right)$. The absolute square of $f\left(t_{D}, \Delta \omega_{A}\right)$ according to (7) was determined via a measurement of the frequency resolved autocorrelation function. In order to account for the phase-modulation of the laser pulses we made the following Ansatz for the phase of the excite and probe function:

$f\left(t, \Delta \omega_{A}\right)=\mid f\left(t, \Delta \omega_{A}\right) \times \exp \left(\mathrm{i} \tilde{\Phi}\left(t, \Delta \omega_{A}\right)\right)$

with

$$
\begin{aligned}
\tilde{\Phi}\left(t, \Delta \omega_{A}\right)= & a_{1}\left(\Delta \omega_{A}\right) t+a_{2}\left(\Delta \omega_{A}\right) t^{2} \\
& +a_{3}\left(\Delta \omega_{A}\right) t^{3}+a_{4}\left(\Delta \omega_{A}\right) t^{4} .
\end{aligned}
$$

For each of the nine experimental curves of Fig. $3 \mathrm{a}$ least-square fit with a specific searching algorithm was used to determine the parameters $a_{1}$ to $a_{4}$ and the peak amplitude of $\left|f\left(t, \Delta \omega_{A}\right)\right|$. For all nine curves of Fig. 3 this procedure gives good agreement of experiment and theory. Especially, the modulation period, the positions of the modulation peaks, the rise of the signal, and the amplitude at $t_{D}=0$ and at later delay times were well reproduced.

With this set of values for $a_{1}\left(\Delta \omega_{A}\right) \ldots a_{4}\left(\Delta \omega_{A}\right)$ the phases of the "excite and probe" function for the nine frequencies $\Delta \omega_{A}$ of the experiment were calculated. Using (5) it can be shown directly that the Fourier transform of the excite and probe function $f\left(t, \Delta \omega_{A}\right)$ at the frequency $\Delta \omega_{A}$ becomes the Fourier transform of the probing laser field $\widetilde{E}_{P}\left(\Delta \omega_{A}\right)$

$$
\widetilde{E}_{P}\left(\Delta \omega_{A}\right) \propto \int d t \exp \left(-\mathrm{i} \Delta \omega_{A} t\right) f\left(t, \Delta \omega_{A}\right) \text {. }
$$

Using the complex function of $f\left(t, \Delta \omega_{A}\right)$ determined above we may now calculate the complex function $\widetilde{E}_{P}\left(\Delta \omega_{A}\right)=\left|\widetilde{E}_{P}\left(\Delta \omega_{A}\right)\right| \exp \left[\mathrm{i} \tilde{\varphi}\left(\Delta \omega_{A}\right)\right]$ for nine frequencies $\Delta \omega_{A}$ by a simple integration. The results are given in Fig. 4. The spectral intensity $I_{p}(\omega)$ $=\left|\widetilde{E}_{P}\left(\Delta \omega_{A}\right)\right|^{2}$ is given by the circles and the phase $\tilde{\varphi}\left(\Delta \omega_{A}\right)$ is given by the triangles (broken curve).

The first important result is the spectral shape of the light pulses. The function $I_{P}(\omega)$ determined from the fitting procedures accurately follows the measured

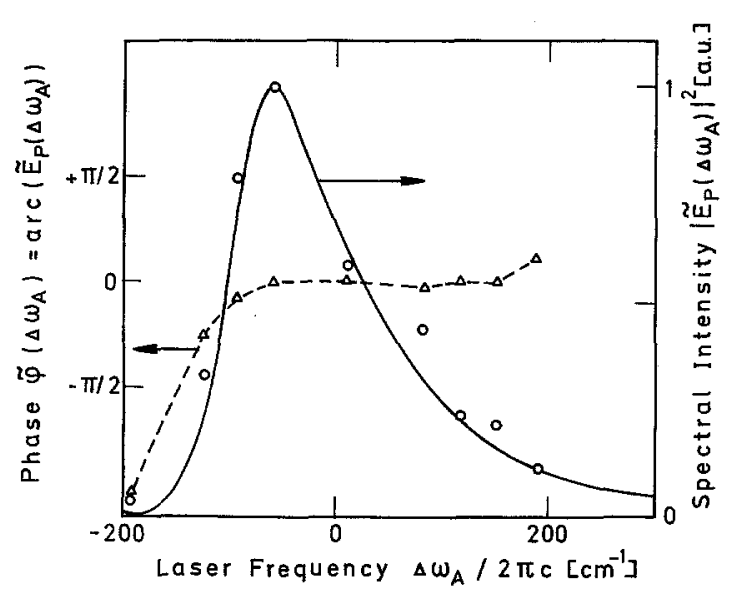

Fig. 4. Calculated (points) and measured (line) spectral intensity and calculated spectral phase (triangles) of the femtosecond laser pulses

spectrum of the light pulses (redrawn from Fig. 1 as solid curve). This fact strongly supports the applicability of the Ansatz used in (9) and the fitting algorithm.

New results can be deduced from the phase $\tilde{\varphi}\left(\Delta \omega_{A}\right)$ of the spectral amplitude $\widetilde{E}_{P}\left(\Delta \omega_{A}\right)$. The slope of $\tilde{\varphi}\left(\Delta \omega_{A}\right)$, i.e., $d \tilde{\varphi} / d \Delta \omega_{A}$, determines the temporal position of the corresponding frequency component, relative to the center of the light pulse (slope zero). From Fig. 4 we learn that the pulse energy in the slowly decaying blue part of the spectrum determines the pulse center, while the red $\left(\Delta \omega_{A}<0\right)$ and the far blue $\left(\Delta \omega_{A}>0\right)$ contributions are found in the trailing wing of the pulse (positive slope). The sharp rise of the spectral intensity in the red part of the spectrum (where the slope of $\tilde{\varphi}$ is positive) indicates that the trailing part of the pulse decays more slowly than the rising part of the pulse. These findings are strongly supported by the frequency resolved autocorrelation function, where increasing widths are found in the low- and in the highfrequency part of the second-harmonic spectrum (Fig. 2).

The temporal properties of the light pulses show up more clearly when the spectral amplitude is Fourier transformed into the time domain to give $E_{P}(t)$ $=\left|E_{P}(t)\right| \exp [\mathrm{i} \varphi(t)]$. Here we face the difficulty of a limited number of data points. Fortunately, the salient features of $E_{P}(t)$ remain independent of the special data handling. The results of the calculation are shown in Fig. 5, where we plotted the pulse intensity (Fig. 5, upper part) and the phase of the light pulses (Fig. 5, lower part) as a function of time. The rising part of the pulse intensity is steeper than the trailing part. There exists a trailing component extending up to $t=250 \mathrm{fs}$. (The modulations superimposed to the signal are artifacts due to the limited number of data points.)

The time dependence of the phase of the light pulses (Fig. 5, lower part) determines the momentary frequency of the pulse via $\omega(t)=-\partial \varphi / \partial t$. 


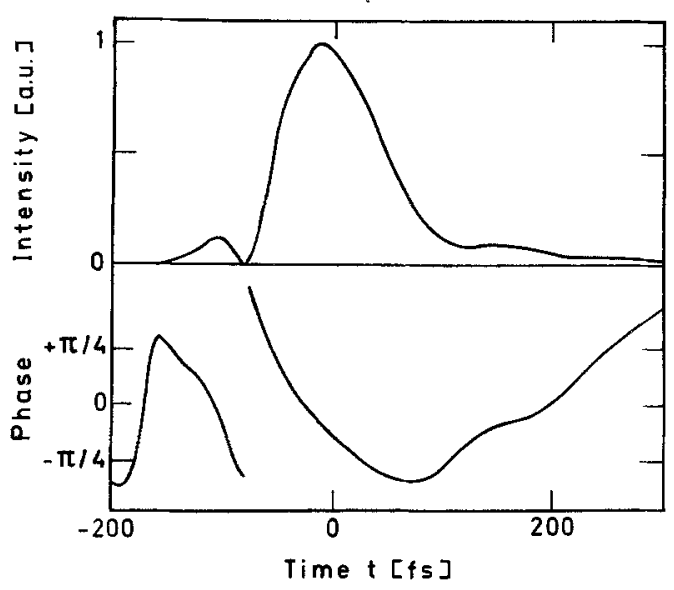

Fig. 5. Calculated intensity $\left|E_{p}(t)\right|^{2}$ and phase angle $\operatorname{arc}\left(E_{p}(t)\right)$ of the femtosecond laser pulses. A pronounced asymmetry of the intensity is found. The time dependence of the phase contains quadratic and higher-order terms

In the rising part of the pulse a positive frequency shift indicates that this part of the pulse is blue shifted. The phase jump at $t=-70 \mathrm{fs}$ is related to the (near) zero of the pulse intensity at the same time and may be a numerical artifact. The blue shift remains until after the peak of the pulse. At later times, during the long trailing part of the pulse intensity, a red-shift is found.

\section{Conclusions}

We have shown that time-resolved coherent Raman scattering may be influenced by the coherence properties of femtosecond light pulses. We have studied the coherent scattering under special experimental conditions, where unexpected phenomena occur which are due to the phase and amplitude properties of the light pulses. A numerical analysis of the experimental data allowed to deduce the time dependence of the amplitude and the phase of the femtosecond light pulses. The salient results are: The femtosecond light pulses are slightly asymmetric with a rapid rising blueshifted part and a more slowly decaying red-shifted part. Our experiments have been performed with femtosecond light pulses from an uni-directional ring dye laser and our results of the pulse properties directly relate to this laser type. It is interesting to note that similar phase and amplitude modulation has been reported in the literature for other femtosecond lasers, e.g., for colliding-pulse mode-locked (CPM) lasers.

Acknowledgement. The authors acknowledge many valuable discussions with Prof. W. Kaiser.

\section{References}

1. R.L. Fork, B.I. Greene, C.V. Shank: Appl. Phys. Lett. 38, 671 (1981)

2. G. Mourou, T. Sizer II: Opt. Commun. 41, 47 (1982)

3. M.C. Nuss, R. Leonhardt, W. Zinth: Opt. Lett. 10, 16 (1985)

4. J. Dobler, H.H. Schulz, W. Zinth: Opt. Commun. 57, 407 (1986)

5. J.A. Vladmanis, R.L. Fork: IEEE J. QE-22, 112 (1986)

6. J.E. Rothenberg, D. Grischkowsky: J. Opt. Soc. Am. B 2, 626 (1985)

7. J.E. Rothenberg: IEEE J. QE-22, 174 (1986)

8. J.M. Diels, J.J. Fontaine, I.C. McMichael, F. Simoni: Appl. Opt. 24, 1270 (1985)

9. Y. Ishida, K. Naganuma, T. Yajima: IEEE J. QE-21, 69 (1985)

10. W.Z. Lin, L.G. Fujimoto, E.P. Ippen: Appl. Phys. Lett. 50, 124 (1987)

11. A. Laubereau, W. Kaiser: Rev. Mod. Phys. 50, 607 (1978)

12. W. Zinth, W. Kaiser: In Ultrashort Light Pulses and Applications, ed. by W. Kaiser, Topics Appl. Phys. 60 (Springer, Berlin, Heidelberg 1988)

13. W. Zinth, M.C. Nuss, W. Kaiser: Chem. Phys. Lett. 88, 257 (1982)

14. N. Kohles, A. Laubereau: Appl. Phys. B 39, 141 (1986)

15. M.D. Levenson, N. Bloembergen: Phys. Rev. B 10, 4447 (1974)

16. R. Leonhardt, W. Holzapfel, W. Zinth, W. Kaiser: Rev. Phys. Appl. 22, 1735 (1987) 